\title{
Criminologie
}

\section{Ville et prison : discours d'hygiénistes réformateurs à Montréal au cours de la deuxième moitié du XIXe siècle}

\section{Martin Dufresne}

Volume 28, numéro 2, 1995

La détention provisoire

URI : https://id.erudit.org/iderudit/017375ar

DOI : https://doi.org/10.7202/017375ar

Aller au sommaire du numéro

Éditeur(s)

Les Presses de l'Université de Montréal

ISSN

0316-0041 (imprimé)

1492-1367 (numérique)

Découvrir la revue

Citer cet article

Dufresne, M. (1995). Ville et prison : discours d'hygiénistes réformateurs à Montréal au cours de la deuxième moitié du XIXe siècle. Criminologie, 28(2), 109-130. https://doi.org/10.7202/017375ar
Résumé de l'article

This study is a discursive analysis which emphasises the notions of moral depravation, urban milieu and prison in the hygienists' discourse. Their knowledge focuses on the human-milieu relationship and is structured in such a way as to promote a vast program of reform. From their standpoint, filth, miasmas, putrefaction, and the cramming together of a massive population with a large contingent of indigents, meant the spread of physical diseases and moral depravation. Packed in the slums, repeatedly entering the local prison for a few days, a segment of the population was at the center of the hygienist preoccupation. Furthermore, the prison was perceived as reproducing defective urban conditions. Reforming both the city and the prison was conceived as a similar project. Reading the social organization as an entity meant that moral depravation is an indicator of the state of society as a whole and therefore should be a concern for everyone. 
VILLE ET PRISON : DISCOURS D'HYGIÉNISTES RÉFORMATEURS À MONTRÉAL AU COURS DE LA DEUXIĖME MOITIÉ DU

XIX' SIÈCLE Martin Dufresne ${ }^{1}$

This study is a discursive analysis which emphasises the notions of moral depravation, urban milieu and prison in the hygienists' discourse. Their knowledge focuses on the human-milieu relationship and is structured in such a way as to promote a vast program of reform. From their standpoint, filth, miasmas, putrefaction, and the cramming together of a massive population with a large contingent of indigents, meant the spread of physical diseases and moral depravation. Packed in the slums, repeatedly entering the local prison for a few days, a segment of the population was at the center of the hygienist preoccupation. Furthermore, the prison was perceived as reproducing defective urban conditions. Re. forming both the city and the prison was conceived as a similar project. Reading the social organization as an entity meant that moral depravation is an indicator of the state of society as a whole and therefore should be a concern for everyone.

Au gré de la pression migratoire et de l'industrialisation à Montréal au cours de la seconde moitié du $\mathrm{XIX}^{\mathrm{e}}$ siècle, une préoccupation à l'égard de l'hygiène physique et morale ralliait un nombre croissant de «éformateurs $^{2}$ ". À leurs yeux, les épidémies de variole et de choléra, les taux de morbidité et de mortalité, enfin la propagation du vice et de l'oisiveté dans des quartiers insalubres étaient les signes des aspects menaçants de l'hypertrophie urbaine et de la civilisation industrielle. L'efficacité de la nation, disaient-ils, devait compter sur le secours d'une science qui poserait la santé physique et morale en termes de déséquilibres des habitudes, du caractère et du milieu.

1. L'auteur prépare une thèse de doctorat en histoire sur la place qu'occupent les représentations du crime et du criminel dans l'évolution du système pénal. Il tient à remercier le Centre de recherche en civilisation canadienne-française de l'Université d'Ottawa pour son appui financier, de même que A. Cellard, P. Tremblay, M. Beaulac, et tout particulièrement $M$. Brosseau, pour leurs commentaires. Criminologie, Université d'Ottawa, 1 Stewart, C.P. 450, Succ. A, Ottawa, ON, K1N 6N5.

2. Je n'entends pas tous les réformateurs, mais bien un certain nombre de ceux qui étaient soucieux de l'hygiène et de la ville à Montréal. Impliqués dans le mouvement hygiéniste, ils exposent leurs points de vue par des conférences, des brochures, les journaux scientifiques et la presse. Médecins pour la plupart, mais aussi ingénieurs, avocats, architectes, chimistes ou autres, ils adhèrent à des associations diverses et occupent parfois des postes dans l'administration publique. Les inspecteurs des prisons, dont il sera question, font partie de ce groupe. 
À l'échelle municipale, puis provinciale, les obstacles à l'adoption de mesures d'hygiène vont progressivement tomber ${ }^{3}$. Parallèlement, le bureau des inspecteurs des prisons, asiles et hôpitaux, établi en 1859 , se chargera, en réaction à la dépravation morale, d'échafauder un vaste plan de réforme des établissements publics. Ce projet d'un réseau d'établissements adaptés à chaque classe de "malheureux » connaîtra lui aussi quelque succès.

La question du crime, ou plus précisément celle de l'immoralité, était constitutive du regard que ces réformateurs portaient sur la ville. Ils faisaient de la moralité une affaire de santé publique, et par là, une dimension clef tant de la connaissance que du bon fonctionnement de l'organisation sociale. C'était, dans le répertoire des outils qui servaient à lire le tout social, faire du crime un indicateur de sa condition ainsi qu'une de ses composantes organiques.

Quant à la prison, cette "école du vice», elle semblait reproduire et accentuer les conditions malsaines de la ville. Si bien que la réforme de l'une et de l'autre s'esquissait dans un espace discursif similaire, et supposait des mesures d'intervention analogues et complémentaires.

Mon intention, ici, n'est pas de chercher à découvrir le non-dit de ces réformateurs-hygiénistes ${ }^{4}$, leurs intentions ou mobiles plus ou moins secrets, ni d'évaluer le succès relatif de leurs interventions. J'étudie plutôt ces liens villeprison en cherchant la cohérence et la convergence de divers discours qui traitent des rapports humain-milieu ${ }^{5}$. Pour ce faire, j'examinerai comment ces

3. Reste que malgré I'établissement d'un Conseil provincial d'hygiène en 1886 , d'autres résistances pèsent sur le champ de la santé publique. Voir M. Farley, P. Keating et $\mathrm{O}$. Keel, «La vaccination à Montréal dans la seconde moitié du $19^{\mathrm{e}}$ siècle : pratiques, obstacles et résistances», dans M. Fournier, Y. Gingras et $O$. Keel (dir.). Sciences \& médecine au Québec, Institut québécois de recherche sur la culture, 1987; M. Farley, $\mathrm{O}$. Keel et $\mathrm{C}$. Limoges, « Les commencements de l'administration montréalaise de la santé publique (1865-1885)", HSTC Bulletin : Journal of the History of Science, Technology and Medicine in Canada, 20 (1982): pp. 24-46, 21 (1982): pp. 85-109. Voir aussi M. Bliss, Montréal au icmps du grand fléau, Montréal, Libre Expression, 1993.

4. Voir M. TÉTREAULT, «L'inégalité sociale devant la mort et la perception de la santé chez les contemporains à Montréal pendant la seconde moitié du XIXe siècle, ou le discours sur la santé publique comme discours idéologique", Nouvelles recherches québécoises, vol. 1, no 2, (1978): pp. 59-81; aussi, C. Pierre-Deschênes, "Santé publique et organisation de la profession médicale au Québec 1870-1918", Revue d'histoire de l'Amérique française, vol. 35, no 3 (1981) : pp. 355-375.

5. J'ai procédé par sondages dans le Journal d'hygiène populaire, dans Public Health Magazine and Literary Review, dans Canada Medical Journal et dans Canada Medical \& Surgical Journal. J'ai étudié les Rapports des inspecteurs des prisons dans les Documents de la Session. Enfin, un éventail de sources diverses, notamment des publications d'auteurs impliqués de près dans la question de l'hygiène et de la réforme urbaine à Montréal, ont été consultées. 
discours cherchent à se faire entendre en se définissant un espace de légitimité, comment sont liés et articulés les divers éléments du savoir qu'ils déploient, comment enfin ils dictent un éventail de mesures d'assainissement.

\section{LA TRIBUNE DU RÉFORMATEUR}

Les réformateurs compilent un éventail d'anomalies que l'observation révèle et qu'indique la statistique sociale ${ }^{6}$. Recourant à l'incitation morale ou encore s'avouant pessimiste dans le but de provoquer, le discours réformateur mise sur le style même pour produire des effets, tout en faisant une ample démonstration du caractère à la fois péremptoire et bien pesé des ses propositions. Jetant çà et là les briques de l'édifice à construire, signalant les progrès accomplis, cernant l'infortune de certaines pratiques, la plume du réformateur considère aussi les progrès des voisins du sud ou des nations européennes. Largement tracé en tenant compte de son auditoire potentiel, tout le discours, dans sa forme, son contenu et son style, vise à provoquer des effets spécifiques. Sensibles à l'ordre social et à la valeur économique de la santé ${ }^{7}$, les réformateurs ambitionnent d'intercéder auprès des élites en faveur du progrès dont tous bénéficieraient inévitablement, puisqu'il y va de l'efficacité de la nation tout entière, de cette "grande lutte des sociétés modernes pour l'existence ${ }^{8}$ ".

Barrières légales et disette financière, esprit corrompu des uns et politique de parti des autres, force est de bien doser les réclamations, qui ne doivent pas passer pour des supplications extravagantes. Raison d'abord, économie et science aussi, sans quoi on n'oserait demander le pécule du progrès. Désintéressé et neutre, éclairé de « la science volant au secours de l'ignorance, faisant ouvre de philanthropie et de nationalité ${ }^{9}$ », l'ami de

6. On réfère à diverses collections de chiffres de la mortalité et de la morbidité. Sur certaines de ces données, voir J.-C. Robert, « The City of Wealth and Death : Urban Mortality in Montreal, 1821-1871 », in W. Mitchison and J. D. McGinnis (ed.). Essays in the History of Canadian Medicine, Toronto, McClelland and Stewart, 1988, pp. 1938; M. Tétreault, «Les maladies de la misère. Aspects de la santé publique à Montréal 1880-1914», Revue d'histoire de l'Amérique française, vol. 36, $\mathfrak{n}^{\circ} 4$ (mars 1983): pp. 507-526. Voir aussi, à partir de 1859, les Rapports du Bureau des inspecteurs des prisons, asiles, etc., dans les Documents de la Session.

7. Le thème est fréquemment abordé dans le Journal d'hygiène populaire. Notamment, voir l'article du docteur A. D. Nicolas, "La valeur économique de la santé ", vol. $1, n^{\text {os }} 7$ et 8 , sept. 1884, pp. 73-76. Voir aussi dans le même journal un extrait de Ja Revue d'hygiène, dans le vol. I, no 11, oct. 1884, pp. 121-123.

8. Circulaire-programme de la société d'hygiène de la province de Québec, 1884 , p. 5. On craint aussi que les épidémies et les taux de mortalité ne freinent l'immigration, à laquelle on associe le développement du pays. Voir Ol. Robitaille et Thos. Blatherwick, Rapport du Bureau central de santé, 1855, pp. 15-16.

9. Le docteur $\mathrm{S}$. Lachapelle dans le Journal d'hygiène populaire, Montréal, vol. I, no I, mai 1884, p. 2. 
l'humanité saura peser les exigences de la liberté et la nécessité de certaines entraves légales. Pour le bien de tous, travailleurs comme patrons, seule la législation peut imposer des standards sans pour autant nuire à la saine concurrence $^{10}$.

Contre ceux qui n'ont pour la science que réticence ou mépris, les réformateurs rappellent que Dieu a aussi créé le corps humain, et que s'il est des coutumes ou des environnements qui gênent son développement, ils s'opposent à sa volonté. Ce serait alors négligence que de s'interdire les bienfaits de la science ${ }^{11}$.

Encore faut-il contrer certaines croyances défavorables au bien-être général dont l'empire et l'étendue défient la mise en place des mesures sanitaires les plus indispensables. L'épidémie de variole qui fauchera quelques milliers de vies à Montréal en 1885-86 rappellera le rempart auquel se butent les visées réformatrices. Bien que l'on fasse mention de l'atteinte aux libertés individuelles ou encore du danger que constituait la vaccination obligatoire, la plupart des observateurs y remarquent la ténacité de certaines croyances populaires. De l'ignorance, disent-il, faisant puissamment obstacle à l'adoption de mesures sanitaires. "L'humanité, diront les insouciants, ne subit pas d'arrêt dans sa course vers sa fin fatale. C'est peine perdue de venir se heurter à la loi inexorable de la nature ${ }^{12}$,"

Les réformateurs n'entendent pas davantage se laisser prendre dans les querelles intestines. Pour certains, les réformes à entreprendre et la classe de la société la plus misérable ne se réduisent pas à des questions confessionnelles ${ }^{13}$. De plus, rappellent-ils, nul n'est totalement à l'abri des miasmes que couve la ville, des émanations putrides que les égouts vomissent dans les habitations ou dans les rues, enfin, des déprédations et de la dépravation que les maisons de charité et les prisons ne suffisent à contenir.

10. $\mathrm{D}^{\mathrm{r}}$ J. I. DESROCHES, «Deux questions vitales : hygiène privée - hygiène publique", Journal d'hygiène populaire, VII a année, no 7, nov. 1890, pp. 193-196; $\mathrm{D}^{\mathrm{r}} \mathrm{D}$. Brochu, Mémoire sur la nécessité d'une inspection hygiénique et médicale des ateliers et des manufactures, Québec, Imprimerie de J. Dussault, 1800, p. 9.

11. Sur le débat, voir $D^{r}$ A. J. H. Crespi, «Sanitary Science from a Religious Point of View», Public Health Magazine and Literary Review, vol. II, $\mathrm{n}^{\circ}$ 6, déc. 1876, pp. 173-181; Henry Howard, M. D., M.R.C.S., Eng., Crime and Insanity: A Paper Read Before the Montreal Medico-Chirurgical Society, by the President, Montreal, The Gazette Printing House, February 7th, 1879, p. 7; J. L. Archambault, Conférence sur l'hygiène générale, Montréal, W. F. Daniel, 1887, p. 5 ; J. T. Read, M. D., The Physical Sufferings of the Poor Viewed from a Sanitary and Sociological Standpoint, Montréal, William Drysdale \& Co., 1898, p. 5 (recueil d'articles qui avaient été publiés dans The Presbyterian Review).

12. J. L. ARCHAMBAULT, op. cit. (1887), p. 5.

13. Care of our Destitute and Criminal Population, Montreal, Salter and Ross, 1857, p. 17 (il s'agit d'un recueil de lettres publiées dans Montreal Gazette et signées «Philanthropy »). 
Bien que l'on fasse des dangers l'affaire de tous, le regard du réformateur est néanmoins fixé sur la pauvreté, pour la placer au cœur du problème urbain. En convergeant vers le personnage du pauvre, ces discours en font à la fois l'objet de l'analyse, des mesures à appliquer et de leur sollicitude. Leur stratégie discursive lui réserve ainsi une place privilégiée, voire fait de lui l'objet d'un parti-pris. C'est en ce sens que le $\mathrm{D}^{\mathrm{r}}$ Desroches, comme d'autres, se permet «une digression en faveur du pauvre ${ }^{14}$. En ce sens aussi, les inspecteurs des prisons, asiles et autres établissements publics disent s'occuper d' « une classe de la société dégradée, il est vraj, mais qui néanmoins mérite nos sympathies, car elle est quelquefois, souvent même, plus malheureuse que coupable ${ }^{15}$ ". Et l'avocat J. L. Archambault, alors président de la Société d'hygiène de la province de Québec, d'ajouter :

Demandons donc à tous ces puissants de la terre moins de dîners qui font les insomnies et les lois mal digérées, de meilleurs égouts et moins de demeures remplies de scrofuleux et de rachitiques, moins de somptueuses constructions pour abriter des chevaux et des pompes à incendie et plus d'abris pour les misères et les haillons de l'humanité souffrante; enfin demandons, s'il est possible, moins de maisons de jeu ou de prostitution et de buvettes qui tuent le corps et vicient l'intelligence et plus de temples à la science et d'écoles du soir où la classe opulente et l'honnête ouvrier viendront puiser la force et la lumière, la santé du corps et la vie de l'âme, seuls biens légitimes et capables de satisfaire ici bas les appétits des sens et les volontés énergiques de notre être ${ }^{16}$.

Le réformateur forge un espace discursif dans lequel se mêlent philanthropie, intérêt public et science, et au nom desquels on ne pourra le prendre à partie. Et du sein même de cet espace, vers lequel convergent divers discours, se dégage une théorie des rapports humain-milieu. Elle repose sur l'équilibre de l'énergie vitale et cherche à saisir ce qui le modifie. Ainsi, la civilisation industrielle et l'hypertrophie urbaine qui l'accompagne creusent des déséquilibres qui affectent tout autant la santé physique que morale. Les conditions de vie montréalaises offrent une prise considérable à la propagation des maladies et à la circulation de l'immoralité. Le milieu

14. Dr J. I. DESROCHES, Quelques réflexions sur le Bureau de santé et sur l'assainissement de Montréal, Montréal, Imprimerie W. F. Daniel, 1887, p. 20. Voir aussi, anonyme, "Our Starving Poor", Public Health Magazine and Literary Review, vol. $1, \mathbf{n}^{\circ}$ 12, June 1876, pp. 376-377.

15. Premier rapport annuel du Bureau des inspecteurs de prisons, asiles, etc., pour les années $1867 \&$ 1868, Documents de la Session no 23, 1869, p. 2 (non-paginé).

16. Conférence... op. cit. (1887), p. 12. Privilégions les enseignements de la science moderne à la poursuite de la fortune et du pouvoir, de dire P. P. Carpenter, dans On the Relative Value of Human Life in Different Parts of Canada (extrait du Canadian Naturalist), Montréal, John Lovell, 1859, p. 1. 
urbain, espace de l'entassement et de la pauvreté, foyer des épidémies, refuge de l'immoralité, sollicite l'attention des réformateurs.

\section{DÉSÉQUILIBRES}

\section{Insalubrité physique et dépravation morale}

Depuis 1884, la Société d'hygiène de la province de Québec fait croisade contre la «falsification industrielle». M. C. A. Pfister, chimiste, exposait les dangers des toxiques employés dans l'industrie et ceux de la falsification alimentaire. Des confitures sucrées au glucose et parfumées de nitrobenzine jusqu'à la "poussière colorante vénéneuse » du papier peint dont on recouvre les murs, on trouvera la source de nombreux maux. La concurrence, bien que saine pour le commerce et l'industrie, force néanmoins le recours à des produits de qualité inférieure, artificiels et potentiellement dangereux lorsque respirés ou absorbés ${ }^{17}$.

Croisade aussi contre l'insalubrité urbaine dont les signes sont légion : remblais d'immondices, odeurs nauséabondes, logements vétustes et cours boueuses au milieu desquelles se trouve parfois une fosse, unique cabinet d'aisance pour de nombreuses familles. L'inefficacité du drainage est telle que les égouts de Montréal ruminent immondices et excréments humains, puis exhalent des gaz délétères qui remontent dans les habitations et dans les rues ${ }^{18}$. On repère ainsi, parmi la saleté et la putrescence, nombre de foyers pestilentiels, sources d'émanation méphitiques qui préfigurent la maladie et la mort.

Tout au long de la seconde moitié du $\mathrm{XIX}^{\mathrm{e}}$ siècle, un mouvement migratoire choisit la ville de Montréal soit comme destination, soit comme étape $^{19}$. Bientôt, l'entassement humain signale avec force les carences et défauts du logement, l'insuffisance des institutions de charité, le problème

17. Circulaire-Programme... op. cit. (1884).

18. A maintes reprises, les imperfections du système de canalisation sont dénoncées, notamment le tunnel de la nue Craig, impropre à l'évacuation. Voir l'ingénieur C. M. Tate, Rapport sur l'assainissement de la cité de Montréal, 1854; W. Kennedy, Report to the Health Commitlee recommending the Re-modeling of the Health Department, Montreal, Lovell Printing \& Publishing Co., 1874; Anonyme, "Sanitary Reports. Public Meeting ", Public Health Magazine and Literary Review, vol. I, n 1, July 1875 , pp. 6-11; l'architecte J. H. Springle, «Ventilated House Drainage », Public Health Magazine and Literary Review, vol. 1, n ${ }^{\circ}$ 10, April 1876, pp. 289-297; le docteur J. I. Desroches, Quelques réflexions... op. cit. (1887).

19. Voir P. A. LINTEAU, R. DUROCHER et J. C. ROBERT, Histoire du Québec contemporain (tome I : De la Confédération à la crise [1867-1929]), Montréal, Boréal, 1989, pp. 164-174. Aussi, J. C. Robert sur la question des «migrations internes », dans "Urbanisation et population : le cas de Montréal en 1861 ", Revue d'histoire de l'Amérique française, vol. $35, n^{\circ} 4$, mars 1982, pp. 523-535. 
du chômage et celui de la promiscuité. Ces maux paraissent d'autant plus alarmants lorsqu'on lie la misère à la maladie et lorsque les épidémies fauchent les vies. Et encore s'étonne-t-on de l'effroyable mortalité infantile pour laquelle chacun avance des estimations 20 .

À travers ces découvertes et les corrélations qu'on élabore, les réformateurs vont façonner leur définition d'un certain problème, leur vérité, celle de la menace sanitaire, et en identifier la source : les quartiers ouvriers. La civilisation moderne marche, et quelque chose d'inévitable et de presque incontrôlable lui fait office de suite : une dégradation du milieu dont la ville est le théâtre. Tous en subissent les effets, mais chez les pauvres, son intensité est alarmante.

C'est qu'on comprend que le miasme humain produit par l'encombrement de la population ouvrière dans des logements exigus, malpropres et insalubres, que les conditions anti-hygiéniques où se passe la vie montréalaise favorisent puissamment la propagation des maladies 21 .

Et, par préoccupation sanitaire, on entend aussi l'état de la moralité. Alors que l'épidémie de choléra qui avait sévi au cours des années précédentes semblait vouloir frapper de nouveau à l'été de 1854, Wolfred Nelson, maire de Montréal, signalait «l'affinité étroite entre la dépravation morale et la dégradation physique 22 ». Et H. H. Miles d'ajouter :

The moral and physical agencies have invariably been found to strenghten and react upon each other. No language can adequately describe the utter degradation of those who, in some of the great centres of national industry, exist in crowded localities when sanitary precautions are wholly neglected, - where there is an entire absence of ash-pits, sewers, drains, and watersupplies, and of ventilation 23 .

20. Voir SIMPSON, J., The Municipal Loan Funds, and the Hospitals and Charities of the Province of Canada, Quebec, Printed at the "Morning Chronicle " Office, $1864, \mathrm{pp}$. XIV et 68 . L'auteur prétend que sur les 825 enfants abandonnés qui étaient à la charge des sœurs de la charité au cours de l'année 1863,602 ont perdu la vie. À ce sujet voir J. C. Robert, "The City of Wealth and Death...», op. cit., p. 31 ; P. Gossage, «Les enfants abandonnés à Montréal au XIX' siècle : la Crèche d'Youville des sæurs grises, 1820-1871 ", Revue d'histoire de l'Amérique française, vol. 40, $\mathrm{n}^{\circ} 4$, printemps 1987, pp. 537-559.

21. J. I. DESROCHES, Quelques réflexions... op. cit. (1887), p. 10.

22. W. NELSON, Notions pratiques sur le choléra, et sur les mesures sanitaires, préservatives et curatives à prendre dans le cas où cette épidémie nous visiterait, Montréal, Imprimerie de Louis Perrault, 1854, p. 4.

23. H. MILES, On the Ventilation of Dwelling-Houses \& Schools, Montreal, Published by John Lovell, 1858, p. 13. Nelson et Miles seront éventuellement membres du Bureau des inspecteurs des prisons. 
En fait, la moralité parle d'elle-même à travers des signes. On trouvera des indications physiques du degré des facultés morales. On connaît pour les tempéraments des caractères physiques et une organisation intellectuelle typiques $^{24}$. Les vêtements loqueteux et l'habitat insalubre parlent aussi de la vertu et servent encore d'indices de moralité. Seulement, les manifestations de l'immoralité se transmettent d'un individu à un autre par contagion, ce qui peut vouloir dire par contact, imitation, enseignement, s'opposant aussi à la conservation de l'équilibre de l'énergie vitale, fondement de la santé parfaite. Les phénomènes qui participent à la contagion charrient des maladies, mais aussi des folies, des immoralités. Et celles-ci semblent progresser au rythme de l'entassement urbain.

Il est pénible d'avoir à constater que malgré les efforts réunis des nombreuses institutions de charité pour mettre des barrières aux vices, la débauche, le vagabondage, l'ivrognerie, la fainéantise et les vices qui en sont les compagnons ordinaires, vont en augmentant dans notre Cité ${ }^{25}$.

Bref, disent les réformateurs, l'intumescence urbaine opère une pression sur le corps et l'esprit de ses occupants. Dans les profondeurs de la ville, dans les ruelles et les logements insalubres de la pauvreté, tels des complices, l'immoralité et la maladie s'expriment, se communiquent. Dans ce «milieu », contagions et miasmes font circuler des maladies physiques et morales.

\section{Les facultês morales et l'énergie vitale}

Là où dénaturation matérielle, putréfaction physique et dépravation morale se rencontrent, l'équilibre de l'énergie vitale se trouve remis en question. Puisque liées indissociablement à la santé physique, les facultés intellectuelles et morales s'affaissent.

L'homme subit dans un milieu malsain une véritable dépression vitale qui doit diminuer le fond d'énergie qu'il possède à l'état de santé parfaite ${ }^{26}$.

A cet égard, des thèses en apparence fort divergentes mènent néanmoins à des constats similaires et suggèrent des interventions rapprochées.

24. Cette typologie fort ancienne distingue les types sanguin, lymphatique, nerveux et bilieux, $\mathrm{D}^{\mathrm{r}}$ Sévérin Lachapelie, La santé pour tous ou notions élémentaires de physiologie et d'hygiène à l'usage des familles, Montréal, Compagnie d'Imprimerie Canadienne, 1880, pp. 188-194. Voir aussi le Dr J. I. Desroches, Catéchisme d'hygiène privée, Montréal, W. F. Daniel, Imprimeur-Éditeur, 1889, pp. 17-23.

25. Rapport du Comité spécial de la maison de correction sur cet établissement et sa direction, Montréal, 4 décembre 1867.

26. Circulaire-Programme... op. cit. (1884), p. 4; voir aussi le $\mathrm{D}^{\mathrm{r}} \mathrm{W}$. Nelson, Notions pratiques... op. cit. (1854), p. 5; H. H. Miles, On the Ventilation... op. cit. (1858), p. 13. 
Le docteur H. Howard, aliéniste, défenseur de la médecine matérialiste, prétend que non seulement les facultés mentales s'incarnent physiquement, mais qu'il en va tout autant des facultés morales.

We find that irritation of the frontal cells, renders the most intellectual man a raving maniac; that irritation of the parietal cells, renders the most honest man a pick-pocket; and that irritation of the cerebral cells, renders the most pure being a filthy, impure creature ${ }^{27}$.

La propension au crime trouve alors un milieu propice à son expression dans les conditions de pauvreté, puisque «darkness and impure air will so destroy the mental organization as to render man an imbecile ${ }^{28}$ ". Le "criminal neurosis", présent chez chacun à différents degrés, s'exprimera plus fortement chez ceux qui sont conçus et élevés dans le crime, la dépravation et la débauche ${ }^{29}$. De sorte que la constitution spécifique de chacun est le fruit de l'hérédité jointe à l'action du milieu.

Par ailleurs, les tenants de la doctrine chrétienne en médecine admettent des différences constitutionnelles et reconnaissent l'effet des lésions cervicales, mais placent néanmoins les facultés de l'intelligence, animées par l'âme, au-dessus du corps. Les états de l'esprit s'y trouvent en quelque sorte liés à la santé physique. Ainsi, la tristesse abaisse l'âme, elle en retire de l'énergie; la colère affaiblit, gonfle les vaisseaux sanguins, nuit à la circulation et provoque parfois une mort subite ${ }^{30}$. Toutes les passions, ces "inclinations violentes " ou "maladies morales [...] dépendent des habitudes, du caractère et du milieu dans lequel on vit ${ }^{31}$ ". Seule la volonté, soutenue par une éducation morale, peut rasseoir les déroutes de l'imagination livrée

27. Dr H. HOWARD, Responsibility and Irresponsibility in Crime and Insanity, A Paper read before the Montreal Medico-Chirurgical Society, (extrait du Canada Medical and Surgical Journal, March, 1879), Montreal, «The Gazette " Printing House, 1879 , p. 6. Sur le débat, voir $\mathrm{D}^{\mathrm{r}} \mathrm{W}$. Osler, «On the Brains of Criminals. With a Description of the Brains of two murderers», Canada Medical \& Surgical Journal, Montreal, X, pp. 385-398, 1881-82, et l'année suivante dans la même revue « Report on the Brains of Richards and O'Rourke », XI, pp. 461-466, 1882-83.

28. Dr H. HOWARD, Mental and Moral Science; with some Remarks upon Hysterical Mania, (extrait du Canada Medical Journal, March, 1878), Montreal, "The Gazette» Printing House, 1878, p. 8.

29. $\mathrm{Dr}^{\mathrm{H}}$. HOWARD, Responsibility and Irresponsibility... op. cit. (1879), p. 8.

30. Dr. S. LACHAPELLE, Manuel d'hygiène d̀ l'usage des écoles et des familles, Montréal, Cadieux \& Derome, 1890, p. 169. Aussi, la peur, en plus de «l'état malsain de la localité » compte pour une des plus importantes causes qui déterminent l'apparition du choléra, selon le docteur W. Nelson, Notions pratiques... op. cit. (1854), p. 10. De même, selon le $D^{r}$ J. I. Desroches, «l'épilepsie se développe fréquemment sous l'influence de la terreur causée par la vue d'une attaque épileptique ", dans Préceptes de l'hygiène scolaire, Montréal, 1891, p. 13.

31. Anonyme, Journal d'hygiène populaire, vol. 1, nº 6, août 1884, pp. 71-72. 
à elle-même et corriger des habitudes malsaines. Il faudra donc, pour la santé de l'adolescent, "surveiller soigneusement les spectacles de tout genre, les tableaux, les images, les lectures, les paroles, les exemples qui seront offerts à son attention ${ }^{32}$ ». En fait, se préserver des désordres, vices et abus par « une vie calme et des mours sévères ${ }^{33}$ ».

Or, d'une perspective à l'autre, le milieu sert à articuler et tout autant à réunir les notions d'hérédité et de volonté. Qu'il s'incarne physiquement ou non, le dérèglement des facultés intellectuelles et morales trouve, dans les milieux pauvres, un terrain fertile. Les inclinations diverses et même la folie s'y rencontrent fréquemment ${ }^{34}$.

L'hygiène a pour but, en modifiant les milieux physiques, de rendre les hommes plus énergiques au travail, instruits, moraux et de les laisser ensuite libres de développer, au sein de la sécurité sociale la plus complète, leur activité laborieuse ${ }^{35}$.

Que de choses ne seraient accomplies grâce à de simples mesures hygiéniques ponctuelles, disent-ils. Ne serait-ce que de se couvrir de vêtements chauds, d'aérer les appartements, de nettoyer éviers et latrines, d'éviter l'amoncellement d'objets nuisibles dans les logements ${ }^{36}$. Éviter aussi l'exténuation, la surcharge émotive, prendre une posture adéquate au travail, bref, ne point privilégier une dimension de l'homme au détriment d'une autre ${ }^{37}$. N'estce pas le cas dans certaines écoles où, plutôt que de doser minutieusement exercice physique et travail mental, on s'évertue à bourrer le crâne des enfants

32. Dr H. GEORGES, «l'hygiène et l'adolescent», Journal d'hygiène populaire, vol. $6, n^{\circ} 6,1889$, pp. 179-181.

33. J. L. ARCHAMBAULT, «Hygiène et morale», Journal d'hygiène populaire, vol. I, n० 16 , janv. 1885 , p. 191.

34. $D^{r}$ J. T. RElD, "Proverty one of the Chief Causes of Insanity", dans, J. T. Reid, The Suffering of the Poor... op. cit. (1898), pp. 19-21.

35. Anonyme, «La statistique", Journal d'hygiène populaire, vol. I, $n^{\circ} 5$, juil. 1884, pp. $51 \cdot 52$.

36. F. P. Mackelcan, Our Health and Our Diseases. Conditions of Montreal in a Sanitary Point of View. We must reform or continue to suffer, Montreal, Lovell Printing and Publishing Company, 1879.

37. Sur l'équilibre de l'éducation du corps et de l'esprit, voir, parmi d'autres, l'article de H. Azaïs, tiré du Journal d'éducation de Bordeaux et publié dans le Journal de l'instruction publique, vol. XII, $\mathrm{n}^{\circ} 6$, juin 1868, pp. 68-70; Dr B. W. Richardson, "Du rôle de la femme comme réformatrice de l'hygiène", reproduit et traduit dans le Journal d'hygiène populaire, vol. $\mathrm{I}, \mathrm{n}^{\mathrm{os}} 2$ et 3 , juin 1884, pp. 16-18; $\mathrm{D}^{\mathrm{r}} \mathrm{H}$. George, «L'hygiène de l'adolescence ", Journal d'hygiène populaire, vol. $6, \pi^{\circ} 6$, nov. 1890 , pp. 178-181. 
de toutes les nouvelles connaissances ${ }^{38}$ ? En fait, vivre une ascèse de l'équilibre physique et mental.

"Pour tout le monde, la manière de vivre doit être réglée, autant que possible, de telle sorte que la fatigue corporelle ou mentale, la faiblesse ou la surexcitation n'en puissent résulter ${ }^{39}$. »

Ainsi, il faut se prémunir contre des affections physiques ou morales en évitant toutes les formes d'excès. Le milieu offre maintes occasions d'abus, de débordements, mais davantage, il fait lui-même figure d'excès. C'est contre lui, parce qu'il est dénaturé, que le gros des réformes doit être déployé.

\section{La dénaturation du milieu}

Le milieu urbain de la civilisation moderne se trouve jugé à l'aune d'une certaine pureté disparue ${ }^{40}$. L'air vicié plutôt que sain, des agglomérations humaines qu'enveloppent des exhalaisons putrides plutôt qu'un étalement humain aéré et désengorgé, l'affaissement de l'énergie plutôt qu'un heureux mélange d'exercice et de repos, l'ennui, voire l'oisiveté, plutôt que la force de caractère et le jugement prompt, forment les couples antithétiques d'un raisonnement qui vise à mettre en évidence la façon dont l'entassement et la misère du milieu conditionnent le miasme de l'immoralité. La prostitution met en évidence ce mal urbain, puisque «les villes sont à peu près exclusivement affectées de cette lèpre 41 ".

Les grandes prisons de Québec et de Montréal donneront aux réformateurs l'occasion de déployer leur discours parce qu'ils y trouvent des maux identiques à ceux de la ville.

Nos districts ruraux ont encore une population si honnête et si morale que leurs prisons sont assez souvent vierges de prisonniers; quand il y en a, le nombre en est si petit qu'il est presque toujours possible de les placer convenablement, par conséquent d'y éviter les dangers et les désordres que l'on voit à Montréal et à Québec ${ }^{42}$.

38. E. A. MEREDITH, Short School Time with Military or Naval Drill, Quebec, Printed by Hunter, Rose \& Co., 1865, pp. 3-4; voir aussi le $\mathrm{D}^{\mathrm{r}} \mathrm{J}$. I. Desroches à propos du surmenage intellectuel, Préceptes de l'hygiène scolaire, Montréal, 1891, p. 10.

39. $\mathrm{D}^{\mathrm{r}} \mathrm{J}$. I. BIBAUD, Quelques considérations sur les causes et l'hygiène des maladies contagieuses et le choléra en particulier, Montréal, Imprimerie du journal «Le Pays», 1866, p. 7.

40. Voir H. H. MILES, On the Ventilation... op. cit. (1858); Dr J. T. Reid, «The Unnaturalness of Life in Large Cities », dans J. T. Reid, The Physical Sufferings... op. cit. (1898), pp. 7-10.

41. Rapport préliminaire du Bureau des inspecteurs d'asiles, prisons, etc., 1859. Documents de la Session, no 32,1860, p. 11.

42. Premier rapporr annuel... op. cit. (1869), p. 14 (non-paginé). 
La prison de Montréal réunit à peu près tous les défauts que les inspecteurs consignent : entassement des détenus, défauts des bâtiments, manques du point de vue de l'hygiène, de la direction, de l'instruction; défauts aussi dans l'organisation qui entravent toute tentative d'y établir une stricte discipline, sans laquelle la réforme morale demeure impossible. Leurs critiques gravitent toutes autour d'un constat indéfectible, celui que l'on y trouve les meilleures écoles du vice. Et l'inspecteur E. A. Meredith d'ajouter, devant la Société littéraire et historique de Québec, «can it be possible that we still have gaols where we are systematically manufacturing criminals instead of reforming them ${ }^{43} »$ ?

Face aux résistances de ceux qui ne voient dans la volonté de réformer le système des prisons et d'opérer sur les individus une réforme durable, que d'incalculables dépenses et des prisons trop luxueuses, les inspecteurs opposent les bénéfices de la réforme aux coûts des déprédations dont la société est victime. Ils rappellent les «droits et [...] devoirs de la société à l'égard des classes malheureuses» et proposent de "se mettre au fait des progrès de la science, profiter de l'expérience des autres, suivre la marche des établissements analogues ailleurs ${ }^{44}$ ».

Leur rapport annuel va s'efforcer de mettre en relief trois dimensions du problème que pose la prison. D'abord, sur le plan administratif, on déplore la pauvreté des registres, voire leur absence, l'insuffísance du personnel et le peu d'uniformité des dépenses. Dans ces conditions, impossibilité de connaître et d'opérer un contrôle sur l'administration des prisons.

Pis encore, l'état des bâtiments. Plusieurs prisons ne disposent toujours pas d'un mur d'enceinte, ce qui prive les détenus de l'exercice physique au grand air. Toute l'architecture s'avère impropre à la surveillance et à l'hygiène. On note les défauts de la disposition des quartiers, les murs rongés par l'humidité, les portes qui ont peine à fermer, et aussi l'air malsain qu'on y respire, l'odeur nauséabonde des latrines et l'endroit souvent mal choisi sur lequel elles reposent.

Enfin, les inspecteurs dénoncent l'effet néfaste de la population flottante qui encombre la prison sur la possibilité d'une réforme morale. Sur les 4021 personnes qui ont franchi la porte de la prison de Montréal une seule fois ou à plusieurs reprises au cours de l'année 1866, 2958 furent

43. E. A. Meredith est alors le Secrétaire du Bureau des inspecteurs. Dans Glance at the Present State of the Common Gaols of Canada, \&c., \&c., \&c., Quebec, Hunter, Rose \& Co., 1864, p. 4.

44. Premier rapport annuel... op. cit. (1869), p. 9 (non-paginé). 
condamnées pour «dérèglement, débauche, oisiveté, etc. ${ }^{45}$ ». De jeunes enfants livrés à la rue; des femmes "presque toutes des prostituées qui y entrent et en sortent une dizaine de fois par année ${ }^{46} »$; des fous que l'on tarde à transférer à l'asile au risque de rendre leur guérison impossible. En somme, des aliénés, des indigents, "et de plus malheureuses créatures encore qu'une première faute, souvent comparativement légère, condamne presque infailliblement de cette sorte à une perte complète ${ }^{47}$ ».

Il y a encore quelque chose de remarquable dans cette prison, c'est le nombre considérable de pauvres qui ont demandé comme une faveur l'avantage d'y être enfermés pour ne pas périr, soit de faim, soit de froid. En hiver, elle est plutôt une maison pour les pauvres qu'un lieu de réclusion pour les criminels 48 .

Que peut-on espérer de ce fourre-tout dans lequel croupissent toutes les catégories de malheureux ? C'est contre cette pratique consistant à réunir de façon inconsidérée tous ces rebuts de la société que les inspecteurs se soulèvent et c'est à partir de celle-ci qu'ils articulent leur projet de réforme. Ce que l'inspecteur Desaulniers ne cessera de dénoncer lorsqu'il écrit le détail de chacune de ses visites. Pour la prison de Saint-Jean : «À ma première visite, elle renfermait 7 prisonniers, 1 femme, 2 jeunes garçons de 12 ans condamnés pour vol et 4 accusés. " Pour celle de Sorel : «Elle contenait 6 prisonniers; sur ces 6 prisonniers, il y avait 2 sourds et muets et un aliéné. » Enfin, à Montréal : «À ma seconde visite, on comptait 310 prisonniers, 153 femmes et 157 hommes; plusieurs jeune enfants avec leur mère et 22 aliénés $^{49}$, 》

45. Journaux de l'Assemblée Législative de la province de Québec, Appendice $n^{\circ}$ 10, vol. 1, Session 1867-68, Québec, Augustin Côté, 1868. Les inspecteurs parviennent à assembler des chiffres semblables pour la prison de Montréal dès 1871. Des 3640 offenses commises par les détenus, 717 comptent pour désordres et vagabondages, 1888 pour ivrognerie, 66 pour folie, etc., voir Cinquième rapport des inspecteurs de prisons et asiles, etc., pour l'année 1872, Documents de la Session $n^{\circ} 31$, 1873, p. 80.

46. Premier rapport annuel... op. cit. (1869), p. 16.

47. Rapport préliminaire, op. cit. (1860), p. 9.

48. Voir le rapport particulier de H. H. Miles, dans Premier rapport annuel... op. cit. (1869), pp. 55-61 (non-paginé). Ses commentaires portent sur la prison de Québec, mais s'empresse-t-il d'ajouter, pour celle de Montréal, «il n'y a pas assez de place pour loger convenablement la population flottante des personnes sans ressources qui viennent y chercher un abri et un refuge". Voir aussi les pages 6 et 7 du même rapport et Rapport préliminaire... op. cit. (1860), p. 5.

49. «Rapport particulier de L. L. L. Desaulniers au Bureau des inspecteurs pour 1869 ", dans Deuxième et troisième rapports des inspecteurs des prisons pour l'année 1869 et en partie pour l'année 1870, Documents de la Session $n^{\circ} 12,1870$, pp. 30-37. 
Cêt arsenal de défauts, sur lequel chaque rapport remet l'accent, empêche l'organisation d'une stricte discipline grâce à laquelle le travail et l'éducation religieuse permettraient d'opérer la réforme morale des détenus. Dans de pareilles conditions, le milieu carcéral amplifie les défauts du milieu urbain; il offre, lui aussi, une prise considérable à la circulation du vice et de l'oisiveté. Des détenus, toutes classes confondues, «s'amusent, $s$ 'instruisent et s'inoculent tous les genres de vices ${ }^{50} »$. En fait, s'y retrouve " un pêle-mêle sans nom de ce qu'il y a de plus dégoûtant et de plus dépravé parmi les rebuts des ruelles les plus infectes de la grande Cité ${ }^{5 i}$ ».

La notion «d'école du vice», attestée par la récidive, et celle de la "population flottante qui s'y complaît " servent non seulement de contenu aux dénonciations des inspecteurs, mais structurent tout autant leur regard et leur parole. Ces rapports, dans lesquels s'exécutent l'observation et la sollicitation, où s'expriment le mal à combattre et la sollicitude pour ces malheureux, fixent la réforme carcérale dans le prolongement de la réforme urbaine. Elle fait partie du mal que l'on condamne, et s'intègre au combat qui s'engage. Pour la prison, comme pour la ville, une population malheureuse s'entasse, donnant ainsi libre cours à la circulation des maladies physiques et morales. Pour chacune, on exige une administration efficace, des moyens proportionnés aux dangers, des registres et des statistiques qui permettent de connaître les maux et d'évaluer le succès des réformes. Pour chacune aussi, on va réclamer des travaux d'infrastructure qui puissent épurer le milieu. Et par une réglementation adéquate, on compte éradiquer les sources du danger et freiner des comportements nuisibles à la santé physique et morale du public.

C'est autour de l'idée de milieu dénaturé que s'étaye le savoir du réformateur. Et par dénaturation, il faut entendre une situation de déséquilibre qu'induit le milieu, des écarts et des conditions dangereuses qui évoquent quelque chose d'inéluctable, mais qui laissent aussi ouverte la possibilité de les modifier, d'en réduire l'intensité. On compte intervenir dans ce milieu et l'on souhaite qu'en contrecarrant son influence, la santé physique et morale s'en porte mieux.

\section{ASSAINIR}

\section{L'épuration du milieu}

Dans l'ensemble, les réformateurs proposent des mesures préventives qui visent à neutraliser le milieu en agissant sur sa structure. Mais il gardent aussi à l'esprit la nécessité de propager les bonnes habitudes contre l'ignorance

50. W. NELSON, cité dans Premier rapport annuel... op. cit. (1869), p. 6 (nonpaginé).

51. Premier rapport annuel... op. cit. (1869), p. 6 (non-paginé). 
et les croyances populaires par des espèces de réflexes sanitaires: Autour des querelles à propos de la vaccination obligatoire et de la question des pouvoirs dévolus au conseil municipal en matière d'hygiène, des associations de citoyens se forment. Rapidement aussi, elles s'essoufflent et se dissolvent. Fondée en 1867, la «Montreal Sanitary Association» semble s'éteindre deux ans plus tard ${ }^{52}$. Elle réapparaît en 1887 et se propose de soumettre à l'attention des autorités toute infraction aux règlements sanitaires. L'association recrute ses membres en leur offrant les services d'un ingénieur qui, une fois l'an, inspectera le drainage, la qualité de l'eau et de la ventilation des habitations ${ }^{53}$. Voit aussi le jour la "Citizen's Public Health Association" en 1875. Sous le patronage du $\mathrm{D}^{\mathrm{r}}$ Hingston, alors maire de la ville, elle compte pousser le conseil municipal à l'action en matière d'hygiène et diffuser des informations par sa revue, le «Public Health Magazine and Literary Review »(1875-187754). Enfin, la Société d'hygiène de la province de Québec (1884) a l'intention de vulgariser le savoir scientifique et d'enseigner les bonnes habitudes sanitaires par le truchement de son Journal d'hygiène populaire.

Régulièrement, on élève la voix pour réclamer un Bureau de santé plus efficace, un personnel permanent et l'adoption de ses recommandations. Pour les réformateurs, il constitue le canal privilégié des mesures préventives et de l'application de la science des médecins hygiénistes, ingénieurs ou chimistes. En dénonçant les sources du mal, les réformateurs font flèche de tout bois contre l'administration municipale et contre des législations inopérantes $^{55}$ : organiser une canalisation adéquate, purger la ville des

52. Voir M. FARLEY, O. KEEL et C. LIMOGES, «Les commencements...", op. cit., notamment les notes 16 et 53 . Les auteurs perdent la trace de l'association en 1869. Voir aussi, dans le même article, les remarques sur l'Association sanitaire de Montréal, p. 36.

53. Voir Constitution, Regulations and By-Laws of the Montreal Sanitary Associatien, Montreal, Printed by Gazette Printing Company, 1887, sur microfilms CIMH $n^{\circ} 01856$; et deux rapports annuels : nos 51079 et 51080 .

54. Voir le «Sanitary Reports. Public Meeting» et «Citizen's Public Health Association », Public Health Magazine and Literary Review, vol. 1, no 1, pp. 6-14. Dans ce même numéro, à la page 18 , le $\mathrm{D}^{\mathrm{r}}$ Larocque prétend qu'il s'agit du quatrième effort d'association de citoyens.

55. Parmi d'autres, Dr J. M. Beausoleil, "Hygiène urbaine ", Journal d'hygiène populaire, vol. 1, no 13, nov. 1884, p. 145 : anonyme, "Sanitary Reports. Public Meeting ", Public Health Magazine and Literary Review, vol. I, n 1, July 1875, pp. 6-11 ; anonyme, "Municipal Mismanagement. The Board of Health », Public Heallh Maga. zine and Literary Review, vol. II, no 3, sept. 1876, pp. 92-95. Voir aussi le texte de H. MacDougall sur la notion de "sanitary idea», "an extremely complex process consisting of investigation, legislation, and administration », dans «Public Health and the «Sanitary Idea» in Toronto, 1866-1890", in W. Mitchison and J. D. McGinnis, Essays... op. cit. (1988), pp. 62-87. 
sources de miasmes, aérer les rues et les logements ; légiférer et appliquer la loi en ce qui a trait à la construction des bâtiments, au travail et à la falsification alimentaire. Certaines habitations sont ces foyers de germes, prétend Mackelcan, et les émanations qu'elles dégagent envahissent les voisins. Bien qu'il faille préserver les libertés individuelles, «it is an act of trespass ", ajoute-t-il, de sorte que les autorités devraient pouvoir pénétrer dans les lieux ${ }^{56}$. Et peut-être aussi faut-il recourir à la loi pour retirer des mains de parents vicieux des enfants voués à la dépravation ${ }^{57}$. Enfin, privilégier l'intervention dans les milieux les plus dégradés, dans les rues infectes, plutôt que l'embellissement des quartiers riches, que l'érection d'un parc d'un coût exorbitant ${ }^{58}$. Plutôt que de donner fière allure à la prison de Québec, d'y gaspiller les fonds publics, tenir compte des directives des inspecteurs relatives à l'architecture et à l'infrastructure des prisons, de sorte qu'on puisse y opérer une réforme morale ${ }^{59}$.

Les réformateurs notent les nombreux défauts qu'ils voient, mais puisque les émanations pestilentielles et les putréfactions constituent une bonne part du danger, l'odorat leur sert aussi de méthode d'enquête. L'odeur signale le danger, permet de le repérer et d'évaluer son potentiel ${ }^{60}$. Ce qui frappe, c'est l'odeur nauséabonde que l'on découvre dans certains quartiers de la ville, la puanteur des prisons attenantes qui envahit les palais de justice, les émanations que dégagent la plupart des lieux d'aisance, enfin l'odeur de la ville tout entière par les chaleurs d'été. En fait, nul ne traite de l'insalubre sans faire part de l'odeur qui a sollicité son attention. On s'intéresse ainsi aux abattoirs, aux cochons que certains engraissent dans leur cour, au nettoyage des rues, aux lieux d'aisance, et on cherche, dans l'usage de la chaux, dans la fumigation, le drainage et autres procédés, les moyens d'éradiquer les odeurs et d'aseptiser le milieu.

56. F. P. MACKELCAN, Our Health and Our Diseases... op. cit. (1879), pp. 2834.

57. Philanthropy, Care of Our Destirute... op. cit. (1857). Notamment les lettres du 17 janvier et du 25 février.

58. Dr J. G. BIBAUD, Quelques considérations... op. cit. (1866), p. 14; Anonyme, Le passé, le présent, l'avenir! Abus, fraudes, injustices, Montréal, 1875.

59. Il semble que les inspecteurs s'étaient efforcés de faire adopter leurs principes de construction des prisons alors qu'on entreprenait la réfection de celle de Québec, Report of the Board of Inspectors of Asylums, Prisons, \& c. for the Year 1860, Sessional Papers $n^{\circ} 24,1861$, pp. 27-28 (non-paginé). Ces efforts semblent avoir été vains puisqu'en 1869, alors qu'elle n'est toujours pas terminée, les inspecteurs laissent croire qu'on a préféré dépenser les fonds disponibles pour l'esthétique, Premier rapport annuel... (1869), p. 7.

60. Sur l'importance du sens de l'odorat et sur la "vigilance olfactive", voir A. Corbin, Le miasme et la jonquille, Flammarion, (1982) 1986. 
Drainer la ville des ordures et des excréments humains qui la pourrissent de l'intérieur afin de conjurer les miasmes. Drainage de la ville par une canalisation adéquate de chacune des demeures, des prisons, des usines et des bureaux, soit de tous les établissements dans lesquels se réunissent plusieurs individus. À cet égard, la question des lieux d'aisance prend une importance capitale. Les inspections des prisons, à la suite d'autres réformateurs, se pencheront sur la question et mettront les latrines à terre sèche à l'essai 61 .

Encore faut-il ventiler et aérer. Les propriétés de l'air sont multiples et tous les reconnaissent. À l'asile, l'air constitue une composante essentielle du traitement contre l'aliénation ${ }^{62}$. On s'interroge sur la meilleure manière de respirer ${ }^{63}$, on redoute les exhalations. On s'efforce de calculer un volume d'air minimum pour chacun et d'en assurer la purification. En matière d'hygiène, on établit des standards, que l'on met à l'essai dans les institutions. À l'asile de Saint-Jean, le docteur H. Howard, surintendant médical, y va de sa propre invention : une petite boîte fixée au châssis qui fait entrer, en le purifiant, un volume d'air de « $26400 \mathrm{pi}^{3}$ par heure, assez pour 12 personnes en santé, et pour six malades dans un hôpital ${ }^{64} »$.

On devra donc, s'il faut bâtir des établissements, s'assurer de la question essentielle de leur aération et tout autant de l'hygiène du site. Selon Desroches, au sujet des écoles :

Le terrain destiné à recevoir le bâtiment scolaire doit être sec ou rendu sec, situé sur une partie élevée et bien aérée, éloigné de tout établissement bruyant, malsain ou dangereux, de tout endroit insalubre ou marécageux, isolé le plus possible de toute habitation 65 .

De même, on exposera le bâtiment de l'ouest au sud-est pour que le soleil exerce son effet bienfaiteur. Même préoccupation en ce qui concerne les prisons. Et s'il faut en bâtir de nouvelles, le choix du site prend une importance capitale. Pour le docteur J. C. Taché, il faut les ériger aux abords de la ville pour éviter les contacts, mais surtout sur un emplacement

61. Premier rapport annuel... op. cit. (1869), pp. 17-28: «Lieux d'aisance » (nonpaginé). Comme le problème du drainage, celui des lieux d'aisance fait couler beaucoup d'encre. Chacun y va de ses observations sur les différentes techniques. La vente de la terre usée comme engrais fait miroiter d'éventuels profits.

62. "Special report of Mr. Taché », dans Report of the Board of Inspectors... op. cit. (1861), pp. 47-48 (non-paginé).

63. La respiration par les narines obtient la faveur, Journal populaire d'hygiène, vol. 1, no 11 , oct. 1884, pp. 123-125.

64. Premier rapport annuel... op. cit. (1869), p. 46 (non-paginé); H. Howard, Ventilation. A Paper read Before the Medical Chirurgical Society, of Montreal, St. Johns; «News» Printing House, 1871.

65. Dr J. I. Desroches, Préceptes... op. cit. (1891), p. 3. 
bien élevé, où le déplacement de l'atmosphère puisse exercer son influence ${ }^{66}$.

En somme, le réformateur foule les trottoirs à l'affût des odeurs dangereuses et des défauts observables. Ses pas le mènent tout droit dans les bas-fonds de la ville, dans des ruelles boueuses où coexistent misère et insalubrité. Les miasmes émanent des matières en décomposition répandues dans les rues et stockées dans les quelques égouts qui sillonnent la ville. Est issu aussi de ces milieux un autre miasme, celui de l'immoralité. Déjà, à la faveur des mesures sanitaires d'infrastructure urbaine, la moralité se redresse, puisqu'elle a une dimension matérielle qu'incarnent l'insalubre, le délabrement, la promiscuité. Premier mouvement de désinfection alors, auquel il faudra ajouter des mesures de régénération. Mais avant d'y arriver, il faut couper la circulation à l'immoralité. Seul remède efficace contre certaines maladies, la séparation doit aussi venir à bout de la contagion morale. D'abord retirer du corps social, puis regrouper selon des critères propres, enfin, régénérer dans un milieu sain.

\section{La circulation de l'immoralité}

La création du Bureau des inspecteurs des prisons suit de quelques années le découpage du territoire du Canada-Est en de nombreux districts judiciaires ${ }^{67}$. On érigera alors des prisons pour chacun des nouveaux districts ${ }^{68}$. S'inspirant des progrès de la science pénitentiaire réalisés ailleurs, les premiers inspecteurs s'empressent d'élaborer un ensemble de règlements afin d'uniformiser le régime carcéral et de faire des prisons de véritables écoles de réforme. Se précisent les rôles et devoirs du personnel, les coûts et quantités concernant le régime alimentaire des détenus, un certain nombre de mesures hygiéniques, et sont mis de l'avant les principes de séparation des détenus par classes. Parallèlement, les inspecteurs préparent un mémoire sur la construction et l'administration des prisons. L'architecture, dont on fournit des plans, doit être favorable à la santé et à la discipline. Contre les

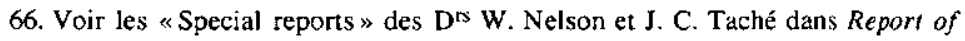
the Board of Inspectors... op. cit. (1861). Les inspecteurs vont se réjouir de la nouvelle prison pour femmes à Montréal, mais, dénoncent-ils, on a érigé la prison sur l'endroit le moins élevé du terrain, dans Septième rapport des inspecteurs des prisons et asiles, pour la province de Québec, 1874, et au juin 30, 1875, Documents de la Session n ${ }^{\circ} 15$, 1875 , pp. 8-9.

67.20 Vict. (1857), c. $28 ; 20$ Vict. (1857), c. 44.

68. Lors de l'institution du Bureau des inspecteurs, en 1859, le Canada-Est dispose des prisons de Montréal, Québec, Trois-Rivières, New Carlisle, Percé, Sherbrooke, Kamouraska et Aylmer. Ces deux dernière voyaient le jour en 1851. À la même époque, treize nouvelles prisons sont en construction pour compléter la réforme des districts judiciaires. À l'exception des prisons de Montréal, Québec, Trois-Rivières et Sherbrooke, le palais de justice fait partie du même édifice que la prison. 
dangers du dépérissement de la santé physique et morale d'une part, et contre ceux de l'isolement complet et prolongé, ils proposent une répartition des détenus par classes et des critères spécifiques d'occupation de l'espace qui vont du choix de l'emplacement à la disposition des pièces ${ }^{69}$.

Enrayer les sources de la corruption morale suppose que l'emprisonnement soit adapté aux caractéristiques des classes de détenus. Entassés dans les prisons communes ${ }^{70}$, ayant souvent l'oisiveté la plus totale pour seule occupation, le vice les corrompt davantage.

2. The inmates of our prisons are made up of individuals differing from each other in age, in sex, in temperament, in physical habits, in religion, in education, in moral and intellectual character, and in their degrees of guilt. 3. It is self evident that the most reformatory prison system would be that which separates from each other the individuals, who would be likely to corrupt each other; and which submits all prisoners either individually or by classes, to such moral and sanitary treatment as is best adapted for their several cases $^{71}$.

C'est pour empêcher la putréfaction morale de cette matière humaine que les inspecteurs vont insister sur la nécessité d'élargir le réseau institutionnel. Il s'agit de contrer la circulation de l'immoralité en s'assurant que jeunes filles et garçons, fous et pauvres, soient dirigés vers des institutions où il serait possible d'établir une stricte discipline. Tout au long de cette deuxième moitié du $\mathrm{XIX}^{\mathrm{e}}$ siècle, les inspecteurs vont répéter inlassablement la nécessité de créer des prisons de division ou prisons centrales sur le modèle des pénitenciers. On y enfermerait les gens condamnés à plus de deux mois de prison, mais aussi les récidivistes, c'est-à-dire ceux qu'on envoie à la prison commune «sept à huit fois par année, à 15 ou 20 jours de détention ${ }^{72}$ ». Après quelques « rechutes», on les emprisonnerait pour une période de deux à trois ans ${ }^{73}$.

69. Report of the Board... op. cit. (1861), p. 23 (non-paginé). Le mémoire fut publié séparément, Memorandum of the Board of Inspectors of Asylums and Prisons. \& c., \& $c$., Quebec, May, 1860. Sur la question de l'isolement complet ou de l'isolement de nuit avec travail en commun mais sous la règle du silence, voir $\mathrm{J}$. Laplante, Prison et ordre social au Québec, Ottawa, Presses de l'Université d'Ottawa, 1989, pp. 111.113.

70. Les critiques des inspecteurs sur l'entassement portent essentiellement sur les deux plus grandes prisons, celles de Québec et Montréal.

71. Memorandum of the Board... op. cit. (1860), p. 1.

72. Premier rapport annuel... op. cit. (1869), p. 15 (non-paginé).

73. Deuxième et troisième rapports... op. cit. (1870), p. 6. Dès le premier rapport du Bureau des inspecteurs, le projet est mis de l'avant. Il en sera question dans tous les rapports, jusqu'à ce qu'ils finissent par admettre, au toumant du siècle, que leur projet restera lettre morte. Sur la question, voir J. Laplante, op. cit. 
La loi qui décréterait l'établissement d'une Maison de Correction, et celle-ci dirigée d'une manière convenable et tout à fait en dehors de la prison commune serait à mon avis, d'une utilité incontestable et rendrait d'incalculables services dans la répression des offenses moindres et de la fréquence la plus ordinaire, telles que vagabondage, ivrognerie, prostitution publique, et les petits larcins, et tendrait, en plus de circonstances qu'on ne se l'imagine, à l'amélioration des mœurs et à la réforme sociale d'un très grand nombre de ces diverses classes de délinquants ${ }^{74}$.

Et bientôt aussi, dès le milieu des années 1870, à l'instar de ce qui se fait en Europe, les inspecteurs semblent se rallier au modèle de l'isolement total pour ces éventuelles prisons de division. Modèle que leurs prédécesseurs avaient pourtant écarté à cause des effets néfastes qu'il produit sur la santé du détenu. Pour le nouvel inspecteur E. Moreau, seul ce système peut éliminer le mal premier de la prison : une «communauté quelconque dans la vie des détenus ${ }^{75}$ ". Bien qu'il en ait été fréquemment question, ni le type de prison ni le modèle de l'isolement complet ne verront le jour. Par ailleurs, quelques établissements viendront compléter le réseau carcéral. Dès 1870 , les inspecteurs voient certains de leurs désirs se réaliser. Outre la maison de réforme de l'île-aux-Noix qui deviendra la Prison de réforme Saint-Vincent-de-Paul, ils pourront compter sur cinq écoles d'industries et de réforme ${ }^{76}$.

Une fois le classement effectué, on mise sur l'éducation religieuse et morale, de même que sur le travail, pour réformer les détenus. Que le travail en question soit productif ou non, il provoque un effet moralisateur puisqu'il a cette double vertu d'exercer le corps et de chasser les mauvaises pensées ${ }^{77}$. Ce qui n'empêche pas les inspecteurs de relier le travail des détenus à la diminution du coût de leur entretien. Et ils s'étonnent de ce que la corporation de la ville de Montréal refuse d'avoir recours aux détenus.

Le nombre des prisonniers qui auraient pu être ainsi employés (sic) au nettoyage des rues et des places publiques, n'est pas assez grand pour nuire

74. Le Recorder Sexton, cité dans Rapport du Comité spécial... op. cit. (1867), p. 4.

75. Rapport particulier de l'inspecteur E. Moreau, Septième rapport... op. cit. (1875), p. 77. Voir aussi le Neuvième rapport des inspecteurs de prisons, asiles, \& c., de la province de Québec pour les années 1876 et 1877. Documents de la Session $\mathrm{n}^{\circ} 23,1878$, pp. 10-11.

76. Deuxième et troisième rapports... op. cit. (1870), p. 38 . On en compte huit dans le Quinzième rapport des inspecteurs des prisons, asiles, etc., de la province de Québec, pour l'année 1884, Documents de la Session n 15, 1884, pp. 6-7.

77. Premier rapport annuel... op. cit. (1869), p. 14. Bientôt, la question du travail va gagner en importance dans les rapports des inspecteurs. Aussi, on se mettra à dénoncer «la routine abrutissante du cassement de la pierre ", Neuvième rapport... op. cit. (1878), P. 12. Voir aussi le Quinzième rapport... op. cit. (1884), p. 19. 
gravement aux ouvriers libres qui, du reste, aurait (sic) eu en échange de ce travail pénible, le cassage de la pierre employée à la réparation des chemins, qui est aujourd'hui cassée par les prisonniers. Quoique sur les 1939 prisonniers condamnés à Montréal dans le cours de l'année 1880,701 fussent des journaliers, nous ne doutons pas qu'une grande partie de ceux qui sont employés aux travaux publics de la ville, sont des gens honnêtes et respectables. Cependant nous devons avouer que la population de la prison de Montréal est surtout recrutée parmi les journaliers qui en forment plus de la moitié. Si le travail des prisonniers dans les rues de la ville devait avoir pour effet d'en faire immigrer un certain nombre et de diriger leur courant sur les comtés ruraux, le mal ne serait pas grand, il y a toujours dans les villes assez de gens qui vivent au jour le jour ${ }^{78}$.

La ligne de démarcation est bien mince entre ceux qui entrent en prison et ceux qui vivent «au jour le jour », qui n'éprouvent pas la régularité du travail. Elle est tout aussi mince entre le regard de celui qui découvre dans la ville le milieu de la maladie physique et de la dépravation morale, et de celui qui retrouve ce même milieu dans les prisons communes. Assainir, c'est corriger soit le milieu, soit les rapports humain-milieu. C'est drainer la ville des eaux fangeuses dont les miasmes pourrissent la santé humaine par une canalisation adéquate, et la drainer aussi de l'immoralité par l'établissement d'un réseau institutionnel qui convienne aux différentes classes de dépravés. Aérer aussi les rues, les habitations, les usines, les prisons, pour éradiquer les odeurs de la maladie et de l'immoralité. Et aérer le milieu urbain en en retirant ces populations flottantes pour une longue période. Enfin, observer les habitudes sanitaires que garantissent l'éducation (religieuse) et le travail régulier. L'assainissement vise la préservation de la santé physique et morale parfaite dans un milieu épuré des écarts, propice à la conservation de l'équilibre de l'énergie vitale. Isolê dans un milieu sain, propre à l'ascèse et à la correction des déséquilibres particuliers à chaque classe de malheureux, le détenu pourra retrouver l'équilibre perdu.

\section{VILLE ET PRISON}

La «civilisation moderne» de ces réformateurs évoque, parfois nostalgiquement, l'évanescence d'un certain monde, mais elle signifie aussi le «devenir », la possibilité, sinon le devoir, d'amender l'organisation sociale, de la rendre plus efficace, de maximiser la somme des énergies humaines qui la composent. Invoquant comme garant de sa neutralité et de sa compétence le savoir scientifique, le discours réformateur insiste sur le caractère impérieux et sur l'autorité de sa parole. Discours provocateur, il constitue

78. Onzième rapport des inspecteurs de prisons, asiles, etc., de la province de Québec, pour l'année 1880, Documents de la Session (non-numérotés), 1882, p. 10. 
un appel à la prise en charge du «devenir». Il se conçoit lui-même comme l'élément dynamique nécessaire d'un monde qui peut maintenant remplir ses promesses. En ce sens, il cultive un certain ordre social et il exige qu'on respecte les règles sur lesquelles on prétend qu'il repose.

Sur le plan du contenu, j'ai insisté sur les thèmes communs à ces discours et sur leur manière de les articuler. S'y dessinent les contours d'une théorie des écarts et des déséquilibres de l'ensemble social. Déséquilibres qui tiennent inévitablement au milieu parce que lui-même est đénaturé, ou encore, qui s'inscrivent dans le rapport entre les humains et le milieu. Dans cette lecture, le phénomène de la dépravation morale et le sort de l'ensemble social sont indissociablement liês.

Enfin, la connaissance que l'on tire de la prison en fait le prolongement de la ville, de même que l'indicateur de son mal. Pourtant, on souhaiterait plutôt qu'elle contribue à relever la ville de quelques-unes de ses misères. Ce point mêrite à mon avis d'être souligné pour le débat sur l'émergence de la prison pénale au XIX ${ }^{\mathrm{e}}$ siècle. Pour J. G. Petit, cette prison est une espèce de «couvent industriel », compromis entre un modèle catholique de la pénitence et une gestion spécifique des populations indigentes qui vise à les mettre au travail ${ }^{79}$. Peut-être faut-il avancer une autre dimension qui, sans rejeter les premières, relèverait du problème urbain et d'une certaine manière d'entrevoir l'effet du milieu sur l'être humain. Estce à dire que comme manière de répartir et d'organiser une population dans un espace, la prison se voudrait l'envers de la ville, un modèle idéal de celle-ci ? Peut-être pas exactement, mais il reste que pour un certain temps, et pour un certain nombre de réformateurs, notamment les hygiénistes, il est possible qu'elle ait été l'esquisse d'un milieu assaini et la tentative pour résoudre un mal essentiellement urbain. Après tout, la prison s'est imposée alors qu'en France et en Angleterre la question urbaine prenait une importance capitale. Alors que, également, les pré-urbanistes ébauchaient leurs projets d'agglomérations humaines entièrement planifiées ${ }^{80}$. Ainsi, le projet carcéral se serait peut-être inscrit, en partie bien sûr, dans un projet plus large, celui de la prise en charge du “devenir" de la ville.

79. J. G. Petit, "Peine et pénitence : les prisons pénales, nouveaux couvents de la civilisation industrielle du $\mathrm{XIX}^{\mathrm{e}}$ siècle », dans B. Gamot (éd.), Histoire et criminalité de l'Antiquité au XXe siècle. Nouvelles approches, Éditions universitaires de Dijon, 1992, pp. $509-517$.

80. Voir notamment F. CHOAY, L'Urbanisme, utopies et réalités, Paris, Seuil, 1965. 